\title{
BOUNDEDNESS OF SIGN-PRESERVING CHARGES, REGULARITY, AND THE COMPLETENESS OF INNER PRODUCT SPACES
}

\author{
EMMANUEL CHETCUTI and ANATOLIJ DVUREČENSKIJ
}

(Received 2 June 2003; revised 3 October 2003)

Communicated by I. Raeburn

\begin{abstract}
We introduce sign-preserving charges on the system of all orthogonally closed subspaces, $F(S)$, of an inner product space $S$, and we show that it is always bounded on all the finite-dimensional subspaces whenever $\operatorname{dim} S=\infty$. When $S$ is finite-dimensional this is not true. This fact is used for a new completeness criterion showing that $S$ is complete whenever $F(S)$ admits at least one non-zero sign-preserving regular charge. In particular, every such charge is always completely additive.

2000 Mathematics subject classification: primary 6C15; secondary 81P10.

Keywords and phrases: Inner product space, orthogonally closed subspace, charge, sign-preserving charge, regular charge, bounded charge, completeness of inner product space.
\end{abstract}

\section{Introduction}

Gleason [4] characterised the set of all $\sigma$-additive states on the system $L(H)$ of all closed subspaces of a real, complex or quaternion separable Hilbert space, $H$, showing that there is a one-to-one correspondence among $\sigma$-additive states, $s$, on $L(H), 3 \leq \operatorname{dim} H \leq \aleph_{0}$, and positive trace operators with unit trace, $T$, on $H$ given by

$$
s(M)=\operatorname{tr}\left(T P_{M}\right), \quad M \in L(H),
$$

where $P_{M}$ is the orthogonal projector from $H$ onto $M$.

In the paper [4], there is an example (see (2.1) below) showing that for any finitedimensional Hilbert space $H$ of dimension at least three, $L(H)$ admits many unbounded charges (= signed measures). The result of Dorofeev and Sherstnev [1] that

(C) 2005 Australian Mathematical Society $1446-7887 / 05 \$ A 2.00+0.00$ 
every $\sigma$-additive measure on $L(H)$ with $\operatorname{dim} H=\infty$ is bounded was therefore very surprising.

In what follows, we show that an analogical result can be extended to signpreserving charges on $F(S)$ with $\operatorname{dim} S=\infty$, that is, for charges $m$ satisfying that if $m\left(M_{i}\right)$ is strictly positive (negative) for a sequence of mutually orthogonal finite-dimensional subspaces $\left\{M_{i}\right\}$, then $m\left(\bigvee_{i} M_{i}\right)$ is not negative (not positive).

We recall that if $S$ is an inner product space over real, complex or quaternion numbers, we can define two families of closed subspaces of $S$.

Let us denote by $F(S)$ the set of all orthogonally closed subspaces of $S$, that is,

$$
F(S)=\left\{M \subseteq S: M^{\perp \perp}=M\right\}
$$

where $M^{\perp}=\{x \in S:(x, y)=0$ for all $y \in M\}$. Then $F(S)$ is a complete lattice with respect to the set-theoretical inclusion [7,2].

Let us denote by $E(S)$ the set of all splitting subspaces of $S$, that is,

$$
E(S)=\left\{M \subseteq S: M+M^{\perp}=S\right\} .
$$

Thus, $E(S)$ is the collection of all subspaces $M$ of $S$ where the projection theorem holds. Observe that every complete subspace is splitting, and $E(S) \subseteq F(S)$. In fact, $S$ is complete if and only if $E(S)=F(S)$ (see [2]).

The paper is organised as follows. A charge on $F(S)$ is a finitely additive mapping. A charge is regular if the value of $m(M)$ for $M \in F(S)$ can be approximated by values on finite-dimensional subspaces of $M$. In Section 2 we characterise $P_{1}(S)$-bounded charges on $F(S)$ - charges bounded on one-dimensional subspaces. In Section 3 we introduce sign-preserving charges, and we show that these are always bounded on all the finite-dimensional subspaces of $S$ whenever $\operatorname{dim} S=\infty$.

In Section 4 we apply this result to obtain a new completeness criterion showing that $S$ is complete if and only if $F(S)$ admits at least one non-zero sign-preserving regular charge. In addition, every such charge is of the form (1.1) for some Hermitian trace operator $T$ (not necessary positive and of trace one), and moreover, such a regular charge is even bounded.

We recall that our completion criterion is not valid for sign-preserving charges on $E(S)$, because every $E(S)$ (also for incomplete $S$ ) admits many regular charges.

\section{2. $P_{1}(S)$-bounded charges on $F(S)$}

A charge on $F(S)$ is any mapping $m: F(S) \rightarrow \mathbb{B}$ such that

$$
m(M \vee N)=m(M)+m(N)
$$


whenever $M, N \in F(S)$ and $M \perp N$. A positive valued charge $m$ such that $m(S)=1$ is said to be a state. A charge $m: F(S) \rightarrow \mathbb{R}$ is a $\sigma$-additive measure or a completely additive measure if $(*)$ holds for any sequence $\left\{M_{n}\right\}$ or any system $\left\{M_{t}\right\}$ of mutually orthogonal elements from $F(S)$. In a similar manner we can define a charge on $E(S)$.

We denote by $P(S)$ and $P_{1}(S)$ the set of all finite-dimensional and of all onedimensional subspaces of $S$, respectively. We say that a charge $m$ on $F(S)$ is

(i) bounded if $\sup \{|m(M)|: M \in F(S)\}<\infty$;

(ii) $P(S)$-bounded if $\sup \{|m(M)|: M \in P(S)\}<\infty$;

(iii) $P_{1}(S)$-bounded if $\sup \left\{|m(M)|: M \in P_{1}(S)\right\}<\infty$.

For example, let $\phi: \mathbb{R} \rightarrow \mathbb{R}$ be a discontinuous additive functional on $\mathbb{R}$ (see for example [5], or [2, Proposition 3.2.4]). Let us define the mapping, $m: L(H) \rightarrow \mathbb{R}$, by

$$
m(M):=\phi\left(\operatorname{tr}\left(T P_{M}\right)\right), \quad M \in L(H),
$$

where $O \neq T \neq k I$ is a Hermitian trace operator on $H, k \neq 0$. Then, for any $H$, $\operatorname{dim} H \geq 3, m$ is an unbounded charge.

In a similar way, now let $0 \neq T \neq k I$ be a Hermitian trace operator on the completion $\bar{S}$ of $S$, where $k$ is a non-zero real constant and $l$ is the identity on $\bar{S}$. The mapping $m: E(S) \rightarrow \mathbb{R}$ defined by

$$
m(M)=\phi\left(\operatorname{tr}\left(T P_{\bar{M}}\right)\right), \quad M \in E(S),
$$

is an unbounded charge on $E(S)$.

A mapping $f: \mathscr{S}(S):=\{x \in S:\|x\|=1\} \rightarrow \mathbb{R}$ is said to be a frame function if there is a constant $W$ (called the weight of $f$ ) such that $\sum_{i} f\left(x_{i}\right)=W$ holds for any maximal orthonormal system (MONS, for short) $\left\{x_{i}\right\}$ in $S$.

The mapping $f: \mathscr{S}(S) \rightarrow \mathbb{R}$ is said to be a frame type function on $S$ if (i) for any orthonormal system (ONS, for short) $\left\{x_{i}\right\}$ in $S,\left\{f\left(x_{i}\right)\right\}$ is summable; and (ii) for any finite-dimensional subspace $K$ of $S, f \mid \mathscr{S}(K)$ is a frame function on $K$.

The following result was originally proved for states in [6], where the first $\sigma$ additive state completeness criterion was presented, and then generalised for charges in [2, Lemma 4.2.1]. In order to be self-contained, we present the proof in details and in a little bit more general form-for $P_{1}(S)$-bounded charges.

LEMMA 2.1. (1) For any $P_{1}(S)$-bounded charge $m$ on $F(S)$ or $E(S), \operatorname{dim} S \neq 2$, there exists a unique Hermitian operator $T=T_{m}: \bar{S} \rightarrow \bar{S}$ such that

$$
m(\operatorname{sp}(x))=(T x, x), \quad x \in \mathscr{S}(S) .
$$

(2) Let $v$ be a unit vector in the completion $\bar{S}$ of $S, \operatorname{dim} S \neq 2$. Then for any $\epsilon>0$ and any $K>0$, there exists $a \delta>0$ such that the following statement holds: If $w \in S$ 
is a unit vector such that $\|v-w\|<\delta$, then for any $P_{1}(S)$-bounded charge $m$ such that the norm of $T=T_{m}$ is less than $K$, and for each finite-dimensional $A \subseteq S$ satisfying the property $v \perp A$, we have the next inequality

$$
|m(A \vee \operatorname{sp}(w))-m(A)-m(\operatorname{sp}(w))|<\epsilon .
$$

PROOF. (1) Suppose that $m$ is a $P_{1}(S)$-bounded charge and define a function $f$ : $\mathscr{S}(S) \rightarrow \mathbb{R}$ via $f(x)=m(\operatorname{sp}(x)),\|x\|=1$. Then $f$ is bounded on $\mathscr{S}(S)$.

Applying the Gleason theorem for finite-dimensional subspaces of $S$, see [2], there is a well-defined bounded bilinear form $t$ such that $f(x)=t(x, x)$ for any $x \in \mathscr{S}(S)$. Hence, $t$ may be uniquely extended to a bounded, bilinear form $\bar{t}$ defined on $\bar{S} \times \bar{S}$. Therefore, there is a unique Hermitian operator $T: \bar{S} \rightarrow \bar{S}$ such that (2.2) holds. We denote by $\|T\|$ the norm of $T$.

(2) Let $\epsilon>0$ and $K>0$ be given. By the continuity of the function $\rho(t)=$ $\left(2-2\left(1-t^{2}\right)^{1 / 2}\right)^{1 / 2}$ we can find a $\delta_{1}>0$ such that $\rho(t)<\epsilon / 2 K$ for any $t \in\left[0, \delta_{1}\right]$.

The continuity of the projection $P_{\mathrm{sp}(v)^{\perp}}: S \rightarrow \mathrm{sp}(v)^{\perp}$, allows us to find a $\delta \in(0,1)$ such that the assumption $\|v-w\|<\delta$ implies $\left\|P_{\mathrm{sp}(v)^{\perp}}(w)\right\|<\delta_{1}$. Fix a $w \in S$ with $\|w\|=1$, and suppose that $A$ is any finite-dimensional subspace orthogonal to $v$. Then $\left\|P_{A}(w)\right\|=\left\|P_{A} P_{\operatorname{sp}(v)^{\perp}}(w)\right\| \leq\left\|P_{\mathrm{sp}(v)^{\perp}}(w)\right\| \leq \delta_{1}$. Thus, we obtain

$$
\left\|\left(I-P_{A}\right)(w) /\right\|\left(I-P_{A}\right)(w)\|-w\|=\rho\left(\left\|P_{A}(w)\right\|\right)<\epsilon / 2 K .
$$

Put $w^{\prime}=\left(I-P_{A}\right)(w) /\left\|\left(I-P_{A}\right)(w)\right\|$. Then we have $\left\|w-w^{\prime}\right\|<\epsilon / 2 K, A \vee \operatorname{sp}(w)=$ $A \vee \operatorname{sp}\left(w^{\prime}\right)$ and $w^{\prime} \perp A$. Calculate

$$
\begin{aligned}
\mid m(A & \vee \operatorname{sp}(w))-m(A)-m(\operatorname{sp}(w)) \mid \\
& =\left|m(A)+m\left(\operatorname{sp}\left(w^{\prime}\right)\right)-m(A)-m(\operatorname{sp}(w))\right| \\
& =\left|m\left(\operatorname{sp}\left(w^{\prime}\right)\right)-m(\operatorname{sp}(w))\right|=\left|\left(T w^{\prime}, w^{\prime}\right)-(T w, w)\right| \\
& \leq\left|\left(T w^{\prime}, w^{\prime}\right)-\left(T w^{\prime}, w\right)\right|+\left|\left(T w^{\prime}, w\right)-(T w, w)\right| \\
& \leq 2\|T\|\left\|w-w^{\prime}\right\|<\epsilon .
\end{aligned}
$$

\section{3. $P(S)$-boundedness of sign-preserving charges}

In the present section we introduce a new kind of charges, sign-preserving charges, and we show that these are always $P(S)$-bounded. We recall that, in general, charges can be unbounded on $F(S)$, as an example below shows. This notion will be applied in the next section to obtain a new completeness criterion for inner product spaces.

We say that a charge $m$ on $F(S)$ is sign-preserving (or we say also that $m$ satisfies the sign-preserving property) if, for any sequence of mutually orthogonal 
finite-dimensional subspaces $\left\{M_{i}\right\}$ of $S$ such that if $m\left(M_{i}\right)>0$ for any $i$, we have $m\left(\bigvee_{i} M_{i}\right) \geq 0$, or $m\left(M_{i}\right)<0$ for any $i$ then $m\left(\bigvee_{i} M_{i}\right) \leq 0$.

It is easy to verify that if $m\left(M_{i}\right)>0$ for any $i$, then

$$
m\left(\bigvee_{i} M_{i}\right) \geq \sum_{i} m\left(M_{i}\right)>0
$$

and if $m\left(M_{i}\right)<0$ for any $i$, we have the opposite inequalities.

For example, every $\sigma$-additive measure $m$ on $F(S)$ or every positive (negative) charge is sign-preserving. Let $H$ be a separable infinite-dimensional Hilbert space and let $m_{1}$ and $m_{2}$ be two different states on $L(H)$ vanishing on all the finite-dimensional subspaces of $H$. Then $m=m_{1}-m_{2}$ is a sign-preserving charge on $L(H)$, and $m$ is neither positive (negative) nor $\sigma$-additive.

On the other hand, let $H$ be a separable Hilbert space with an ONB $\left\{x_{n}\right\}_{n=1}^{\infty}$. Define the state $m_{1}(M)=\sum_{n=1}^{\infty} 1 / 2^{n} m_{x_{n}}(M), M \in L(H)$, and let $m_{2}$ be any finitely additive state on $L(H)$ vanishing on all the finite-dimensional subspaces of $H$. Then $m=: m_{1}-m_{2}$ is a bounded charge on $L(H)$ which is not sign-preserving. Indeed, let $M=\bigvee_{n=2}^{\infty} \operatorname{sp}\left(x_{n}\right)$. Then $m\left(\operatorname{sp}\left(x_{n}\right)\right)=1 / 2^{n}$ for any $n$, but $m(M)=1 / 2-1=-1 / 2$. More general, if $m_{1}$ is a state defined by (1.1) and $m_{2}$ as above, then $m=m_{1}-m_{2}$ is a bounded charge which is not sign-preserving.

Let now $s$ be a state on $L(H)$ vanishing on all the finite-dimensional subspaces of $H$. According to [3], the range of $s$ is the whole interval $[0,1]$. Take an arbitrary discontinuous additive functional $\phi$ on $\mathbb{R}$. Then the mapping $m$ on $L(H)$ defined by $m(M)=\phi(s(M)), M \in L(H)$, is a sign-preserving charge vanishing on all the finite-dimensional subspaces of $H$ which is unbounded on $L(H)$.

We recall that according to [7, Lemma 33.3],

(1) $F(S)$ is an atomic, complete lattice with orthocomplementation satisfying the exchange axiom (that is, if $M$ is an atom of $F(S), N \in F(S), M \nsubseteq N$, then $M \vee N$ covers $N$ (that is, if $N \subseteq C \subseteq M \vee N$ for some $C \in F(S)$, then $C \in\{N, N \vee M\}$ );

(2) if $M \in F(S)$ and $x \in S$ is a non-zero vector, then $M \vee \operatorname{sp}(x)=M+\operatorname{sp}(x) \in$ $F(S)$;

(3) $\bigwedge M_{i}=\bigcap_{i} M_{i}$ for any system $\left\{M_{i}\right\}$ from $F(S)$.

LEMMA 3.1. Let $S$ be an inner product space and let $N$ be a subspace of $S$, $\operatorname{dim} N=n \geq 1$. Then

$$
F\left(N^{\perp}\right)=\left\{A \in F(S): A \subseteq N^{\perp}\right\}, \quad E\left(N^{\perp}\right)=\left\{A \in E(S): A \subseteq N^{\perp}\right\} .
$$

Proof. If $X \subseteq S$, then $X^{\perp^{\perp}}:=\left\{x \in N^{\perp}: x \perp X\right\}$. Let $\operatorname{dim} N=1$ and suppose $A \in F(S)$ and $A \subseteq N^{\perp}$. Then $A^{\perp_{N}^{\perp} \perp_{N}^{\perp}}=\left(A^{\perp} \cap N^{\perp}\right)^{\perp_{N^{\perp}}}=\left(A^{\perp} \cap N^{\perp}\right)^{\perp} \cap N^{\perp}=$ $\left(A^{\perp \perp} \vee N\right) \cap N^{\perp}=(A+N) \cap N^{\perp}$. Since $N$ is an atom of $F(S)$ and $A \subseteq N^{\perp}, N \nsubseteq A$, 
we have that $(A+N) \cap N^{\perp}$ covers $A$, while $A \subseteq(A+N) \cap N^{\perp} \subseteq A+N$. Hence, $(A+N) \cap N^{\perp}=A$, that is, $A \in F\left(N^{\perp}\right)$.

Conversely, if $A \in F\left(N^{\perp}\right)$, then $A^{\perp_{N}^{\perp} \perp_{N \perp}}=\left(A^{\perp \perp}+N\right) \cap N^{\perp}=A$. The exchange axiom implies $A^{\perp \perp}=\left(A^{\perp \perp}+N\right) \cap N^{\perp}=A$, that is, $A \in F(S)$.

The general case of $\operatorname{dim} N=n>1$ can be obtained by $n$-times repeating the case $\operatorname{dim} N=1$.

Let now $A \in E(S)$ and $A \subseteq N^{\perp}$. If $x \in N^{\perp}$, then $x=x_{A}+x_{A^{\perp}}$, where $x_{A} \in A$ and $x_{A^{\perp}} \in A^{\perp}$ so that $x-x_{A}=x_{A^{\perp}} \in A^{\perp_{N}}$ which gives $A+A^{\perp_{N}}=N^{\perp}$ and $A \in E\left(N^{\perp}\right)$.

Conversely, let $A \in E\left(N^{\perp}\right)$. Then $A+A^{\perp_{N}}=N^{\perp}$ and $A+A^{\perp_{N}}+N=N^{\perp}+N=S$. If $a \in A$ and $u \in A^{\perp_{N}}, v \in N$, then $(a, u+v)=0$, that is, $A^{\perp_{N}}+N \subseteq A^{\perp}$. If now $x \in A^{\perp}$, then $x=x_{A}+x_{A^{\perp_{N}}}+x_{N}$ which gives $x_{A}=0$, that is, $A^{\perp} \subseteq A^{\perp_{N}}+N$.

Therefore, if $\operatorname{dim} N=n \geq 1, N \subseteq S$, then any charge $m$ on $F(S)(E(S))$ can be restricted by Proposition 3.1 to a charge $m_{N^{\perp}}$ on $F\left(N^{\perp}\right)\left(E\left(N^{\perp}\right)\right)$ by $m_{N^{\perp}}(M)=m(M)$ if $M \in F\left(N^{\perp}\right)$.

If $\operatorname{dim} S<\infty$, then it can happen that $m$ is unbounded. In what follows, we show that if $\operatorname{dim} S=\infty$, then every sign-preserving charge on $F(S)$ is $P_{1}(S)$-bounded as well as $P(S)$-bounded. We will follow the basic ideas of Dorofeev-Sherstnev [1] (see also [2, Theorem 3.2.20]), who proved an analogical result for the frame-type functions.

Let us recall that if $H$ is a Hilbert space, then by a self-adjoint operator on $H$ we mean always an operator $A$ defined on a subspace, $S$, of $H$ which is dense in $H$.

Inspiring that, let us denote by $\operatorname{SPC}(H)$ the set of all $P_{1}(S)$-unbounded signpreserving charges defined on $F(S)$, where $S$ is an arbitrary dense subspace of $H$.

Our aim is to show that $\operatorname{SPC}(H)=\emptyset$.

LEMMA 3.2. Let $\operatorname{SPC}(H) \neq \emptyset, \operatorname{dim} H=\infty$. There exist a dense subspace $S$ of $H$ and a charge $m \in \operatorname{SPC}(H)$ on $F(S)$ such that, for any one-dimensional subspace $N$ of $S$ with $|m(N)|>1$, we have $m_{N^{1}} \notin \operatorname{SPC}\left(N^{\perp_{H}}\right)$.

Proof. If $\operatorname{dim} N<\infty$, then $N^{\perp}$ is dense in $N^{\perp_{H}}$, where ${ }^{\perp_{H}}$ denotes the orthocomplementation in $H$, and a sign-preserving charge on $F(S)$ is also a sign-preserving charge on $F\left(N^{\perp}\right)$.

Suppose that the assertion does not hold. Then, for any dense subspace $S$ of $H$, for any charge $m \in \operatorname{SPC}(H)$ on $F(S)$, there exists a one-dimensional subspace $N_{1}$ of $S$ with $\left|m\left(N_{1}\right)\right|>1$ such that $m_{N_{1}^{\perp}} \in \operatorname{SPC}\left(N_{1}{ }^{\perp_{H}}\right)$.

Since $H$ is an infinite-dimensional Hilbert space, it is isomorphic with its subspace $N_{1}^{\perp_{H}}$. Consequently, any charge from $\operatorname{SPC}\left(N_{1}^{\perp_{H}}\right)$ also does not fulfil the hypothesis. In particular, for $m_{N_{1}}$ and we can find a one-dimensional subspace $N_{2}$ of $N_{1}^{\perp}$ with $\left|m\left(N_{2}\right)\right|>1$ such that $m_{\left(N_{1} \vee N_{2}\right)^{\perp}} \in \operatorname{SPC}\left(\left(N_{1} \vee N_{2}\right)^{\perp_{H}}\right)$. 
Continuing this process by induction, we find a sequence of mutually orthogonal subspaces $\left\{N_{n}\right\}$ of $S$ such that $\left|m\left(N_{n}\right)\right|>1$ and $m_{\left(N_{1} \vee \cdots \vee N_{n}\right)^{\perp}} \notin \operatorname{SPC}\left(\left(N_{1} \vee \cdots \vee N_{n}\right)^{\perp_{H}}\right)$ for any $n \geq 1$.

There are infinitely many $n$ 's such that $m\left(N_{n}\right)>1$ or $m\left(N_{n}\right)<-1$. Without loss of generality, we can assume that all $m\left(N_{n}\right)$ have the same sign.

Denote by $A=\bigvee_{n} N_{n}$. In the first case, for any integer $n \geq 1$, we have

$$
\begin{aligned}
m(S)=m\left(A^{\perp}\right)+m(A) & =m\left(A^{\perp}\right)+\sum_{i=1}^{n} m\left(N_{i}\right)+m\left(\bigvee_{i=n+1}^{\infty} N_{i}\right) \\
& \geq m\left(A^{\perp}\right)+\sum_{i=1}^{n} m\left(N_{i}\right) \geq m\left(A^{\perp}\right)+n
\end{aligned}
$$

when we have used the sign preserving property of $m$, which gives a contradiction.

In a similar way we deal with the second case.

LEMMA 3.3. Let $\operatorname{SPC}(H) \neq \emptyset, \operatorname{dim} H=\infty$ There exists $m \in \operatorname{SPC}(H)$ and $a$ one-dimensional subspace $X_{0}$ of $S, S$ dense in $H$, such that

$$
\max \left\{\left|m\left(X_{0}\right)\right|, \sup \left\{|m(Y)|: Y \in P_{1}\left(X_{0}^{\perp}\right)\right\}\right\}=1 .
$$

PROOF. Take $m$ from Lemma 3.2 and multiplying $m$ by some non-zero constant, if necessary, we obtain (3.2).

Since the proofs of the following two lemmas are identical with those in [2, Lemma 3.2.18] and [2, Lemma 3.2.19], they are omitted.

LEMMA 3.4. Let $m \in \operatorname{SPC}(H), \operatorname{dim} H=\infty$, satisfy the condition of Lemma 3.3. Then there exist orthonormal vectors $e_{1}, e_{2}, e_{3} \in S, S$ being the dense subspace of $H$, such that $\left|m\left(\operatorname{sp}\left(e_{i}\right)\right)\right|>1$ for any $i=1,2,3$.

LEMMA 3.5. Let $H$ be a real four-dimensional Hilbert space. Let $e_{1}, e_{2}, e_{3}, e \in$ $\mathscr{S}(H)$ such that $e_{1}, e_{2}, e_{3}$ are mutually orthogonal, and $e \notin\left\{e_{1}\right\}^{\perp} \cup\left\{e_{2}\right\}^{\perp} \cup\left\{e_{3}\right\}^{\perp}$, be given. Then there exist two non-zero vectors $x$ and $y$ in $H$ such that

(1) $e=x+y$;

(2) $\left(x, e_{1}\right)=\left(y, e_{2}\right)=(x, y)=\left(y-\|y\|^{2} e, e_{3}\right)=0, y-\|y\|^{2} e \neq 0$.

We recall that a closed subset $R$ of a complex or quaternion Hilbert space $H$ which is a manifold with respect to the real field $\mathbb{R}$ is said to be completely real if the inner product $(\cdot, \cdot)$ from $H$ takes real values on $R \times R$. Equivalently, if and only if there is an orthonormal set $\left\{e_{j}\right\}$ in $R$ such that $R$ is the closure of the real linear combinations of the $e_{j}$. 
PROPOSITION 3.6. Any sign-preserving charge on $F(S), \operatorname{dim} S=\infty$, is $P_{1}(S)$ bounded.

Proof. Suppose the converse, that is, let $\operatorname{SPC}(H) \neq \emptyset$, and let $m \in \operatorname{SPC}(H)$ satisfy (3.2). Let us set $f(x):=m(\operatorname{sp}(x)), x \in \mathscr{S}(S)$. Select orthonormal vectors $e_{1}, e_{2}, e_{3}$ from Lemma 3.4 with $\left|f\left(e_{i}\right)\right|>1, i=1,2,3$, and define the constant

$$
C=\max _{1 \leq i \leq 3}\left\{\left|f\left(e_{i}\right)\right|, \sup \left\{|f(x)|: x \in \mathscr{S}\left(\left\{e_{i}\right\}^{\perp}\right)\right\}\right\} .
$$

From the unboundedness of $f$ it follows that there is a vector $h \in \mathscr{S}(S)$ such that $|f(h)|>3 C$. It is clear that $h \notin \bigcup_{i=1}^{3}\left\{e_{i}\right\}^{\perp}$ and put $\lambda_{i}=\left(h, e_{i}\right) /\left|\left(h, e_{i}\right)\right|, i=1,2,3$. Then $\left(h, \lambda_{i} e_{i}\right)$ is real for $i=1,2,3$. Let $M$ be a completely real subspace of dimension 4 containing $h$ and all $\lambda_{i} e_{i}$ 's.

Applying Lemma 3.5 to vectors $\lambda_{i} e_{i}$ 's and $h$, we find two non-zero vectors $x$ and $y$ in $M$ such that

$$
\left(x, \lambda_{2} e_{2}\right)=\left(y, \lambda_{3} e_{3}\right)=(x, y)=\left(z, \lambda_{1} e_{1}\right)=0, h=x+y,
$$

where $z=y-\|y\|^{2} h$ is a non-zero vector. Since $\operatorname{sp}\{z, h\}=\operatorname{sp}\{x, y\}=\operatorname{sp}\{y, h\}$, we have $f(h)+f(z /\|z\|)=f(x /\|x\|)+f(y /\|y\|)$. From the construction we conclude that $z \in\left\{e_{1}\right\}^{\perp}$, so that $|f(z /\|z\|)| \leq C$. Similarly, $|f(x /\|x\|)|,|f(y /\|y\|)| \leq C$. Since $|f(h)| \leq|f(h)+f(z /\|z\|)|+|f(z /\|z\|)|$, then

$$
|f(h)+f(z /\|z\|)| \geq|f(h)|-|f(z /\|z\|)|>3 C-C=2 C,
$$

we finally obtain from the last equality

$$
2 C \geq|f(x /\|x\|)+f(y /\|y\|)|=|f(h)+f(z /\|z\|)|>2 C,
$$

which is a desired contradiction.

THEOREM 3.7. Any sign-preserving charge on $F(S), \operatorname{dim} S=\infty$, is $P(S)$-bounded. Moreover, there is a unique Hermitian trace operator $T$ on $H$ such that

$$
m(\operatorname{sp}(x))=(T x, x), \quad x \in \mathscr{S}(S) .
$$

PROOF. In view of Proposition 3.6, $f(x):=m(\operatorname{sp}(x)), x \in \mathscr{S}(S)$, is bounded. Therefore, by (1) of Lemma 2.1, there is a Hermitian operator $T$ on $\bar{S}$ such that $f(x)=(T x, x), x \in \mathscr{S}(S)$.

We now show that $T \in \operatorname{Tr}(H)$. If $T=0$, the statement is evident. Let now $T \neq 0$ and suppose $T \notin \operatorname{Tr}(H)$. Then there is an ONS $\left\{f_{1}, \ldots, f_{n_{1}}\right\}$ in $H$ such that $\sum_{k=1}^{n_{1}}\left|\left(T f_{k}, f_{k}\right)\right|>1$. Choose an $\epsilon>0$ such that $\sum_{k=1}^{n_{1}}\left|\left(T f_{k}, f_{k}\right)\right|>1+\epsilon$. It is 
easy to see that for $\left\{f_{1}, \ldots, f_{n_{1}}\right\}$ we can find an ONS $\left\{h_{1}, \ldots, h_{n_{1}}\right\}$ in $S$ such that $\left\|h_{k}-f_{k}\right\|<\epsilon /\left(2 n_{1}\|T\|\right), k=1, \ldots, n_{1}$. Then

$$
\begin{aligned}
\left|f\left(h_{k}\right)-\left(T f_{k}, f_{k}\right)\right| & \leq\left|\left(T\left(h_{k}-f_{k}\right), f_{k}\right)\right|+\left|\left(T h_{k}, h_{k}-f_{k}\right)\right| \\
& \leq 2\|T\|\left\|h_{k}-f_{k}\right\|<\epsilon / n_{1},
\end{aligned}
$$

so that

$$
\sum_{k=1}^{n_{1}}\left|f\left(h_{k}\right)\right| \geq \sum_{k=1}^{n_{1}}\left|\left(T f_{k}, f_{k}\right)\right|-\sum_{k=1}^{n_{1}}\left|\left(T f_{k}, f_{k}\right)-f\left(h_{k}\right)\right|>1 .
$$

Put $H_{1}=\left\{h_{1}, \ldots, h_{n_{1}}\right\}^{\perp_{H}}$, then $S_{1}=H_{1}$ is a dense subspace in $H_{1}$, so that, $m \mid F\left(S_{1}\right)$ is a sign-preserving charge on $F\left(S_{1}\right)$. Therefore, as in the beginning of the present proof, there is a Hermitian operator $T_{1}\left(=P_{H_{1}} T P_{H_{1}}\right.$ ) on $H_{1}$ such that $f(x)=\left(T_{1} x, x\right)=(T x, x), x \in \mathscr{S}\left(S_{1}\right)$. Here $T_{1}$ is not any trace operator since $T \notin \operatorname{Tr}(H)$.

Repeating the same reasonings as above, we find an ONS $\left\{f_{n_{1}+1}, \ldots, f_{n_{2}}\right\}$ in $H_{1}$ such that $\sum_{k=n_{1}+1}^{n_{2}}\left|\left(T f_{k}, f_{k}\right)\right|>1$, and we find an ONS $\left\{h_{n_{1}+1}, \ldots, h_{n_{2}}\right\}$ in $S_{1}$ with $\sum_{k=n_{1}+1}^{n_{2}}\left|f\left(h_{k}\right)\right|>1$. Continuing this process, we find a countable family of orthonormal vectors $\left\{h_{1}, h_{2}, \ldots\right\} \subset S$ and a sequence of integers, $\left\{n_{i}\right\}_{i=0}^{\infty}, n_{0}=0$, such that $\sum_{k=n_{i-1}+1}^{n_{i}}\left|f\left(h_{k}\right)\right|>1$, for any $i \geq 1$, which gives $\sum_{k=1}^{\infty}\left|f\left(h_{k}\right)\right|=\infty$.

Without loss of generality, we can assume that all $f\left(h_{n}\right)>0$ or $f\left(h_{n}\right)<0$. Set $A=\bigvee_{n} \operatorname{sp}\left(h_{n}\right)$. In the first case, for any $k \geq 1$,

$$
m(S)=m\left(A^{\perp}\right)+\sum_{i=1}^{k} \sum_{j=n_{i-1}+1}^{n_{i}} m\left(\operatorname{sp}\left(h_{j}\right)\right)+m\left(\bigvee_{i>n_{k}} \operatorname{sp}\left(h_{i}\right)\right) \geq m\left(A^{\perp}\right)+k,
$$

which is a contradiction. In a similar way we deal with the second case. Therefore, $T \in \operatorname{Tr}(H)$, and this proves that $m$ is $P(S)$-bounded.

\section{Sign-preserving regular charges and completeness criterion}

In this section, we present a new completeness criterion showing that $S$ is complete if and only if $F(S)$ admits at least one non-zero sign-preserving regular charge. This result extends measure-type completeness criteria given, for example, in [2, Section 4.3.2].

We say that a charge $m$ on $F(S)(E(S))$ is regular if, given $M \in F(S)(M \in E(S))$ and given $\epsilon>0$, there is a finite-dimensional subspace $N$ of $M$ such that

$$
\left|m\left(M \cap N^{\perp}\right)\right|<\epsilon .
$$

THEOREM 4.1. An inner product space $S$ is complete if and only if $F(S)$ admits at least one non-zero sign-preserving regular charge. 
Proof. The necessity is evident. Suppose, therefore, that $S$ is an infinite-dimensional inner product space, and let $m$ be a non-zero sign-preserving regular charge. According to Theorem $3.7, m$ is $P(S)$-bounded. Let $T$ be a Hermitian operator from (2.3).

Let $B$ be an arbitrary orthogonally closed subspace of $S$ and let $\left\{e_{i}\right\}$ be any MONS in $B$ and define $B_{0}=\left\{e_{i}\right\}^{\perp \perp}$. Then $B_{0} \subseteq B$. We claim that $B_{0}=B$.

We see that

$$
m\left(B_{0}\right)=m\left(B_{0}\right)+m\left(B \cap B_{0}^{\perp}\right)=m\left(B_{0}\right)+1-m\left(B^{\perp} \vee B_{0}\right)=m(B)
$$

(which is true for any charge $m$ on $F(S)$ ).

If we had $B_{0} \neq B$, then $\bar{B}_{0} \neq \bar{B}$, and we can find a unit vector $v \in \bar{B}$ which is orthogonal to $\bar{B}_{0}$. There exists a unit vector $e \in S$ such that $m(\operatorname{sp}(e)) \neq 0$. Indeed, there exists $M \in F(S)$ such that, say, $m(M)>0$. Given $M$, we find a sequence $\left\{M_{n}\right\}$ in $P(S)$ of non-decreasing subspaces of $M$ such that $m(M)=\lim _{n} m\left(M_{n}\right)$. Without loss of generality we can assume that $m(\operatorname{sp}(e))>0$. Applying Lemma 2.1 to $\epsilon=m(\operatorname{sp}(e)) / 3>0$ and to $v \in \bar{B}$, we can find a $\delta>0$ such that, for any unit vector $w \in B$ with $\|w-v\|<\delta$ and any $A \perp v, \operatorname{dim} A<\infty$, we have (2.4) for every $P_{1}(S)$-bounded charge $s$ on $F(S)$ for which $\left\|T_{s}\right\|=\|T\|$.

Define a unitary operator $U: S \rightarrow S$ such that $U e=w$ and $U f=f$ for any $f \perp e, w$. Then $m_{U}$ defined via $m_{U}(M)=m\left(U^{-1}(M)\right), M \in F(S)$, is a $P_{1}(S)$ bounded, regular charge on $F(S)$ for which $\left\|T_{m_{U}}\right\|=\|T\|$.

Hence, for $B$ there exists a sequence $\left\{B_{n}\right\}$ of finite-dimensional subspaces of $B$, $B_{n} \subseteq B_{n+1}$ for $n \geq 1$, such that $m_{U}(B)=\lim _{n} m_{U}\left(B_{n}\right)$.

We assert that $m_{U}(B)=\lim _{n} m_{U}\left(B_{n} \vee \operatorname{sp}(w)\right)$.

Calculate,

$$
\left|m_{U}\left(B_{n} \vee \mathrm{sp}(w)\right)-m_{U}(B)\right| \leq\left|m_{U}\left(B_{n} \vee \mathrm{sp}(w)\right)-m_{U}\left(B_{n}\right)\right|+\left|m_{U}\left(B_{n}\right)-m_{U}(B)\right| .
$$

We now follow the ideas and symbols from the proof of (2) of Lemma 2.1 with norm $\|T\|$ less than a constant $K>0$. Let $\epsilon>0$ be given. Set

$$
w_{n}^{\prime}=\left(I-P_{B_{n}}(w)\right) / \|\left(I-P_{B_{n}}(w) \| .\right.
$$

Then $\left\|w-w_{n}^{\prime}\right\|<\epsilon / 2 K, B_{n} \vee \operatorname{sp}(w)=B_{n} \vee \operatorname{sp}\left(w_{n}^{\prime}\right)$, and $w_{n}^{\prime} \perp B_{n}$. Hence,

$$
\begin{aligned}
\mid m_{U} & \left(B_{n} \vee \operatorname{sp}(w)\right)-m_{U}\left(B_{n}\right) \mid \\
& =\left|m_{U}\left(\operatorname{sp}\left(w_{n}^{\prime}\right)\right)\right|=\left|\left(T_{m_{U}} w_{n}^{\prime}, w_{n}^{\prime}\right)\right| \\
& \leq\left|\left(T_{m_{U}} w_{n}^{\prime}, w_{n}^{\prime}\right)-\left(T_{m_{U}} w_{n}^{\prime}, w\right)\right|+\left|\left(T_{m_{U}} w_{n}^{\prime}, w\right)-\left(T_{m_{U}} w, w\right)\right| \\
& \leq\left\|T_{m_{U}}\right\|\left\|w_{n}^{\prime}\right\|\left\|w_{n}^{\prime}-w\right\|+\left\|T_{m_{U}}\right\|\left\|w_{n}^{\prime}-w\right\|\|w\| \leq \epsilon .
\end{aligned}
$$

Consequently, $m_{U}(B)=\lim _{n} m_{U}\left(B_{n} \vee \operatorname{sp}(w)\right)$, and by $(\star), m_{U}(B)=m_{U}\left(B_{0} \vee \operatorname{sp}(w)\right)$. 
Therefore, given $\epsilon>0$ there is an integer $n_{0}$ such that for any $n>n_{0}$

$$
m_{U}\left(B_{n} \vee \operatorname{sp}(w)\right)-\epsilon<m_{U}\left(B_{0} \vee \operatorname{sp}(w)\right)<m_{U}\left(B_{n} \vee \operatorname{sp}(w)\right)+\epsilon
$$

and

$$
m_{U}\left(B_{0}\right)-\epsilon<m_{U}\left(B_{n}\right)<m_{U}\left(B_{0}\right)+\epsilon .
$$

Using these inequalities and (2.4), we get

$$
\begin{aligned}
m_{U}\left(B_{0}\right) & =m_{U}\left(B_{0} \vee \operatorname{sp}(w)\right)>m_{U}\left(B_{n} \vee \operatorname{sp}(w)\right)-\epsilon \\
& >m_{U}\left(B_{n}\right)+m_{U}(\operatorname{sp}(w))-2 \epsilon>m_{U}\left(B_{0}\right)+m(\operatorname{sp}(e))-3 \epsilon=m_{U}\left(B_{0}\right),
\end{aligned}
$$

which contradicts the beginning and the end of former inequalities, and this proves $B_{0}=B$.

Due to the arbitrariness of $B \in F(S)$, we conclude that $F(S)$ is orthomodular. The criterion of Amemiya and Araki [2, Theorem 4.1.2], yields that $S$ is complete, as claimed.

THEOREM 4.2. Any sign-preserving regular charge on $F(S)$ of an inner product space $S, \operatorname{dim} S=\infty$, is completely additive, and there is a trace operator $T$ on $\bar{S}$ such that $m(M)=\operatorname{tr}\left(T P_{M}\right), M \in F(S)$. In addition, the regular charge is always bounded.

PROOF. If $m$ is a zero function, the statement is trivially satisfied. Suppose that $m$ is a non-zero sign-preserving regular charge.

According to Theorem 4.1, $S$ is a Hilbert space, and due to (i) of Lemma 3.2, there is a Hermitian operator $T$ on $S$ such that $(T x, x)=m(\operatorname{sp}(x))$ for any unit vector $x \in S$. Moreover, by Theorem 3.7, $T$ is a trace operator on $S$.

Express $T=T^{+}-T^{-}$, where $T^{+}$and $T^{-}$are positive and negative parts of $T$. Let $S^{+}, S^{-}$and $S_{0}$ be the subspaces of $S$ generated $\left\{x_{i}: \lambda_{i}>0\right\},\left\{x_{i}: \lambda_{i}<0\right\}$, and $\left\{x_{i}: \lambda_{i}=0\right\}$, respectively, where $T=\sum_{i} \lambda_{i}\left(\cdot, x_{i}\right) x_{i}$. Then, for any unit vector $x \in S^{+}, m(\operatorname{sp}(x))>0$ and, for any unit vector $y \in S^{-}, m(\operatorname{sp}(y))<0$. Therefore, $m\left(S^{+}\right)=\lim _{n} m\left(S_{n}\right)$, where $S_{n} \subseteq S_{n+1}$ are finite-dimensional subspaces of $S^{+}$. Hence, $m\left(S^{+}\right) \geq \sum_{i} m\left(\operatorname{sp}\left(x_{i}\right)\right)$ for any ONB $\left\{x_{i}\right\}$ in $S^{+}$which implies $m\left(S^{+}\right)=\operatorname{tr}\left(T^{+}\right)$. In a similar way, we have $m\left(S^{-}\right)=-\operatorname{tr}\left(T^{-}\right)$. Since $m\left(S_{0}\right)=0$, we have $m(S)=\operatorname{tr}(T)$.

If now $M$ is an arbitrary subspace of $F(S)$, then $T_{M}$ is the restriction of $P_{M} T P_{M}$ onto $M$, where $P_{M}$ is the orthogonal projector of $S$ onto $M$, is a trace operator. We repeat the above reasoning for $T_{M}$. Hence, $m(M)=\operatorname{tr}\left(T_{M}\right)=\operatorname{tr}\left(T P_{M}\right), M \in F(S)$.

It is easy to show that the mapping $M \mapsto \operatorname{tr}\left(T P_{M}\right), M \in F(S)$, is a completely additive function on $F(S)$ and bounded. 
We recall that Theorem 4.1 does not hold for the case of $E(S)$. Indeed, let $x$ be a unit vector in $S$. The mapping $m_{x}(M)=\left\|x_{M}\right\|^{2}, M \in E(S)$, where $x=x_{M}+x_{M^{1}}$ and $x_{M} \in M, x_{M^{\perp}} \in M^{\perp}$, is a regular charge on $E(S)$ for any complete or incomplete $S$.

We conclude the article with some comments.

(1) We recall that we do not know whether any regular charge on $F(S)$ is signpreserving.

(2) If a regular charge is $P_{1}(S)$-bounded, then Theorem 4.1 holds for any $P_{1}(S)$ bounded regular charge.

(3) We do not know whether every regular charge on $F(S)$ with $\operatorname{dim} S=\infty$ is $P_{1}(S)$-bounded. This is unknown even if $S$ is a Hilbert space.

\section{Acknowledgements}

The authors are indebted the referee for his valuable remarks which improved the readability of the paper.

The paper has been supported by the grant VEGA 2/3163/23 SAV, Bratislava, Slovakia.

\section{References}

[1] S. V. Dorofeev and A. N. Sherstnev, 'Functions of frame type and their applications', Izv. Vyssh. Uchebn. Zaved. Mat. 4 (1990), 23-29 (in Russian); translation in Soviet Math. 34 (1990), 25-31.

[2] A. Dvurečenskij, Gleason's theorem and its applications (Kluwer Acad. Publ., Dordrecht, Ister Science Press, Bratislava, 1992).

[3] A. Dvurečenskij and P. Pták, 'On states on orthogonally closed subspaces of an inner product space', Letters Math. Phys. 62 (2002), 63-70.

[4] A. M. Gleason, 'Measures on the closed subspaces of a Hilbert space', J. Math. Mech. 6 (1957), 885-893.

[5] G. Hamel, 'Eine Basis aller Zahlen und die unstetigen Lösungen der Funktionalgleichung: $f(x+y)=f(x)+f(y)^{\prime}$, Math. Anal. $60(1905), 459-462$.

[6] J. Hamhalter and P. Pták, 'A completeness criterion for inner product spaces', Bull. London Math. Soc. 19 (1987), 259-263.

[7] F. Maeda and S. Maeda, Theory of symmetric lattices (Springer, Berlin, 1970).

Mathematical Institute

Slovak Academy of Sciences

Štefánikova 49

SK-814 73 Bratislava

Slovakia

e-mail: chetcuti@mat.savba.sk,dvurecen@mat.savba.sk 